\title{
Current Technology in Advancing Medical Education: Perspectives for Learning and Providing Care
}

\author{
Joshua Moran $^{1} \cdot$ Gregory Briscoe ${ }^{2} \cdot$ Stephanie Peglow ${ }^{2}$ \\ Received: 18 January 2018 / Accepted: 25 May 2018 / Published online: 13 June 2018 \\ (C) Academic Psychiatry 2018
}

Healthcare and medical training have no immunity to universal, rapidly changing technology. In medical education, advances like simulations, virtual patients, and e-learning have evolved as pedagogical strategies to facilitate an active, learner-centered teaching approach. According to Chhetri et al., contemporary generations of trainees have grown up immersed in various technologies and are now less functional in the traditional classroom setting [1]. Yet, not all of today's medical trainees or educators are equally adept and comfortable with technology. Educators are tasked with selecting and filtering appropriate technology-based curricula [1].

Advancement in education requires discerning which learning-assisting technologies merit usage in specific scenarios. To better improve patient care in contemporary times, continual innovative efforts between psychiatric educators and trainees remain essential for fully exploiting technology's potential. The objective of this commentary is to discuss the various available medical training technologies and subsequent perceptions of trainees to these modalities. Additionally, this commentary considers how educationbased technologies could improve or hinder the learning experience of medical trainees.

\section{Methods}

We conducted a review of articles published from 2007 to 2018 utilizing an online literature search with PubMed and Google Scholar, as well as a professional medical library search via the EVMS Brickell Medical Science Library System using the following key phrases: medical education

\section{Stephanie Peglow}

peglowsl@evms.edu

1 Edward via College of Osteopathic Medicine, Blacksburg, VA, USA

2 Eastern Virginia Medical School, Norfolk, VA, USA or medical students and technology, e-learning, simulators, virtual reality, mobile devices, audience participation, computer based, medical illustration, or social media. To identify relevant systematic review articles, MeSH terms were also employed in the search to include these additional termsmedical students, attitude to computers, technology, computers, computer-assisted instruction, computer simulation, simulation training, internet, medical education, medical illustration, computer based, or curriculum. A second search was created to include multimedia use in medical lectures; search terms included the following: medical education, medical students and audiovisual aids, medical illustration, or educational technology. Additionally, the following MeSH terms were included in the second search: medical education, medical students, attitude to computers, multimedia, audiovisual aids, computer-based, or curriculum.

Inclusion and exclusion criteria for eligibility were established; no formal scoring system was employed. Systematic reviews written in English between the years of 2007-2018 were deemed eligible via inclusion criteria if written to inform medical educators on the fields of undergraduate medical training, postgraduate medical training, or continued medical education. An article met exclusionary criteria if only contributing redundant information seen in other (included) systematic reviews or otherwise failed to meet the above conditions. One author (GB) screened titles and abstracts of candidate articles to isolate studies which met the inclusion criteria; selected articles were then reviewed more thoroughly to analyze content.

To organize data from each systematic review meeting inclusion criteria, contributing authors read each review study in its entirety and subsequently consolidated article details into a shared Google Docs table. Chosen systematic reviews were organized into the following medical education topics of interest: e-learning, multimedia in lectures, technology-assisted audience participation, virtual reality and simulators, mobile devices, and social media. Systematic reviews not contributing to any of these specific technology-associated topics were 
deleted from our analysis. To appraise the quality and design of eligible systematic reviews, each review article was itemized based on outcome measures, review population studied, study purpose, results, identified conclusions, and reported or observed review limitations.

\section{Results}

Database searches using our key terms of interest yielded 958 articles. As planned, one author scanned each title and abstract of searched articles to compile a list of 81 relevant systematic reviews (those passing inclusion criteria and escaping exclusion criteria). Sixty-two of the articles passing inclusion criteria failed to fit into any of our six-addressed technologyassociated topics of interest; so, these articles were excluded. Of the articles passing inclusion criteria, 19 reviews were relevant to the following technology-associated educational modalities: 4 reviews on e-learning, 1 review on multimedia, 6 reviews on virtual patients and simulators, 3 reviews on audience response systems, 2 reviews on mobile devices, and 3 reviews on social media. No systematic reviews were found specific to multimedia usage in medical education lectures. Three of the smaller systematic reviews in the virtual patient and simulators category were assessed to only contribute information redundant to the other three larger systematic reviews; therefore, these smaller reviews were excluded. Our review appraisal yielded a final 16 articles to be included in our reflection on medical education technology.

A few exceptions were made for this commentary regarding cited articles. First, a non-systematic review (a brief literature review) was included in the introductory background information to provide a contemporary perspective on technological opportunities in medical education. Secondly, no systematic reviews were found specifically addressing the use of multimedia in medical education; therefore, a single review was included to reveal how media has altered the trainee's experience in medical school classroom. Lastly, the Accreditation Council for Graduate Medical Education (ACGME) guidelines were included to address how the conclusion of our commentary may affect current and future postgraduate residency training in psychiatry.

\section{E-Learning}

Flexibility and active learning methods take precedence in contemporary medical education. E-learning - a web-based technology that extends teaching past the classroom - permits learners to hear and engage educators in lieu of or in addition to traditional classroom lectures. To formulate an effective elearning course, collaboration must occur among course directors, teaching faculty, and technology experts.
Despite time and fiscal costs of initial creation, e-learning curricula offer a platform for easy tracking of trainee improvement in knowledge and performance mastery. E-learning helped clinicians circumvent geographic and scheduling restraints and therein promote participation in continuing medical education [2]. Web-based learning was perceived as most valuable when associated with real-time feedback, self-assessments, simple interface, extended time for completion, and topic relevance [3, 4]. E-learning interventions that are perceived as too cursory and lacking relevance or interactivity are viewed less favorably [2]. A study interviewing orthopedic surgery trainees found that e-learning not only accelerated the learning of psychomotor skills but also offered superiority in cost-effectiveness, learner satisfaction, and self-directed pace and focus [5]. These qualities make e-learning ideal for exposing trainees to rare and complex medical scenarios. Such training interventions reinforce recognition of clinical patterns and orchestrate trainee reflection on key training points [5].

\section{Multimedia}

Various technological media categories have been employed to enhance the presentation of medical science topics to trainees. A meta-analysis of 266 studies conducted in 2010 revealed that $89 \%$ of web-based medical training courses included paragraph-form static written text, in addition to multimedia tools like videos, diagrams, and pictures [6]. Multimedia (such as tutorials and diagrams) and interactive self-assessments (such as patient cases, quizzes, or other feedback) were incorporated into over half of e-learning courses. Videos simultaneously fuse both auditory and visual information. Videos engage various areas of the trainee's cognition during lectures [7]. Video-based lectures enable trainees to harness repetition, self-paced practice, and active learning. As with e-learning, trainees benefit the most from videos containing self-assessments, integrated lecture objectives, images, lecture PowerPoint slides, limited duration ( $<15 \mathrm{~min}$ ), quality design, and reputable featured lectures [7]. In fact, multimedia transforms the role of medical educators from that of hosting formal lectures to that of leading discussions and creatively maximizing trainee comprehension via media intervention tools [7].

\section{Technology in Audience Participation}

Audience response systems (ARS) technology has been increasingly utilized to stimulate more active learning in the classroom. ARS may facilitate student in-classroom participation and encourage group problem solving (depending on how the ARS is integrated into the experience) [8]. Anonymity in responses allows the learner to engage without fear of embarrassment or being singled out by peers or the instructor [9] Regarding the incorporation of ARS into curricula, learners 
report strong positive acceptance, increased attentiveness, and enhanced engagement and enjoyment of the lecture experience. One controlled study suggested that immediate feedback after questions (as facilitated by ARS) may improve knowledge condensation [10]. Unfortunately, ARS have shown weak or equivocal results in long-term knowledge retention and learning outcomes; these inconclusive results have impaired academic institutional implementation of the ARS technology [8].

\section{Virtual Reality and Simulations}

As in the military and aerospace industry, medicine has helped to pioneer the use of simulators and virtual reality. To enhance knowledge application, educators have developed virtual patient (VP) encounters (realistic, animated clinical scenarios portraying a broad array of pathologies) to exercise the medical decision-making skills. Virtual reality has been frequently employed by procedural specialty trainees to improve skill development.

Research has yielded mixed reviews of the efficacy of simulations in medical training. A meta-analysis involving various medical professionals across 4 controlled trials compared simulation-based interventions to non-technological interventions [11]. Except for 1 trial, each of the other 3 trials revealed that high-fidelity simulations lacked superiority in areas of trainee confidence, performance, and knowledge [11]. In contrast, two meta-analysis studies comparing simulations to other non-technology-based interventions concluded that simulations yielded significant advantages in knowledge improvement, skill mastery, time to skill acquisition, and trainee satisfaction $[12,13]$.

If designed and selected properly, simulation usage may bring specific advantages to medical education. Trainees have identified feedback, opportunities for repeated practice, realism, and team-focused communication skills as predictive variables contributing to a simulation's success rate and acceptance. Although data regarding actual patient outcome improvement was not found, the use of simulators in medical education appears effective in engaging a medical trainee in active learning [13].

\section{Mobile Devices}

Mobile devices have evolved to accommodate the numerous demands of the highly mobile clinician and trainee. As of $2006,85 \%$ of healthcare providers have adopted mobile devices in patient care [14]. Smartphones enable trainees the ability to multitask, while instantly refreshing knowledge on diagnoses, medical management, patient health information, medical calculations, or the most contemporary literature [15]. Now able to receive real-time, point-of-care computation, trainees can employ idle time and maximize learning by utilizing web-based study material and current literature. Mobile device apps offer improved accessibility to clinical literature, continued medical education, and error prevention tools [14, 15]. Additionally, these devices also permit faster clinical communication and subsequent response times to patient's needs [14]. Mobile devices remain limited in areas of battery life, malware risks, potential privacy breaches, or erroneous information in searches. Trainees express concern about smartphone usage appearing disrespectful to patients, attendings, or coworkers [15]. No current studies exist regarding mobile devices improving actual patient outcomes $[14,15]$.

\section{Social Media}

Due to its prevalence, social media represents a potentially valuable tool for educators. As of 2012, 14.2\% of the world's population were active Facebook users; such estimates speak to the global prevalence of social media usage [16]. Medical students who blogged exhibited improved knowledge, empathy, exam scores, and reflective writing skills but failed to improve test scores [17]. Social media sites can function as a platform for students to exchange advice and medical information throughout their healthcare training [18]. Technical issues, variable participation levels, and privacy or security concerns were primary factors that thwarted full acceptance of blogging by medical students and educators $[16,17]$. Most learners described positive overall reactions to social media and blogging interventions [17]. Additionally, social media creates an avenue through which mental health professionals might be able to educate the public on mental health topics. Medical professionals must be taught and reminded to abstain from unprofessional conduct and privacy breaches while utilizing social media sites [16].

\section{Discussion}

Increasingly integral to the practice of medicine, technology endeavors to streamline a clinician's work and to offer credible, easily accessible information. To enhance trainee growth and empower innovative scientific leaders, educators should play a crucial role in how technology transforms medical education. Trainees prefer technology-associated modalities that offer learning material that is interactive, reputable, simple, pragmatic, and coupled with relevant feedback. Innovations like virtual reality and simulations effectively increase knowledge, performance skills, and team communication through realistic clinical cases. Educators utilize social media to promote student reflection and to address difficulties that trainees experience. The ACGME tasks psychiatry residencies with guiding trainees to employ technology to attain the following milestones: self-reflection and assimilation of relevant information for informed decision-making [19].

Educators must consider whether the benefits of added flexibility and real-time feedback implemented by technology- 
assisted learning outweigh the downsides of the social isolation associated with classroom-independent learning. This potential decline in camaraderie sparks some of medical educators' support for implementing blogs and social media into curricula. In the presence of technology overload, educators must innovate curriculum that bolsters each trainee's humanistic touch. Additionally, medical educators may advocate testing basic technological competencies in the Medical College Admission Test before matriculation or requiring continued education on technologies throughout medical training.

With the amount and variety of technology-based resources ballooning, educators receive expanding opportunity to create and modify new training techniques. Medical educators must instruct trainees how to consistently find the most pertinent, trustworthy, and contemporary information. Medical educators in psychiatry may enhance psychiatric training education by discovering ways to incorporate virtual reality into curricula. For example, educators could consider programming virtual patients and simulators to mimic psychotropic medication side effects, delirium, or drug intoxications. As artificial intelligence evolves, trainees may utilize virtual patients for psychotherapy skill development. Continued research into how integrated technology affects the trainee's attitude and patient care outcomes remains vital for future advancement.

This commentary does not strive for exhaustive assessment or concluding judgment concerning each educational technology. Most reviews looked at studies of single-site implementation of educational outcomes, and few had non-technology controls; hence, the quality of the evidence was limited. Additionally, our literature search unearthed no actual patient outcome measures regarding each incorporated medical technology. Numerous factors, ranging from practice setting, personal preferences, and treatment population, affect how each trainee and physician perceives and adopts technological changes in medicine. Therefore, conclusions made from the experience of medical trainees cannot be generalized to all generations of practicing physicians. Further research into technology usage among different generations and practice types would isolate the most ideal technology-related training goals. Such innovation could help foster development of the best practice models for technology training in undergraduate and postgraduate medical education.

Acknowledgements The authors would like to thank Esther May Sarino, MLIS, for her expert help in crafting and refining the literary search.

\section{Compliance with Ethical Standards}

Ethical Considerations IRB approval was not necessary due to this being a review of literature. EVMS IRB reviewed the proposal and determined it to be not human subjects research (IRB \# 17-12-NH-0272).

Disclosure On behalf of all authors, the corresponding author states that there are no financial or personal conflicts of interest.

\section{References}

1. Chhetri S. E-learning in neurology education: principles, opportunities and challenges in combating neurophobia. J Clin Neurosci. 2017;44:80-3.

2. Cook D, Steinert Y. Online learning for faculty development: a review of the literature. Med Teach. 2013;35(11):930-7.

3. Wong G, Greenhalgh T, Pawson R. Internet-based medical education: a realist review of what works, for whom and in what circumstances. BMC Med Educ. 2010;12 https://doi.org/10.1186/14726920-10-12.

4. Maertens H, Madani A, Landry T, Vermassen F, van Herzeele I, Aggarwal R. Systematic review of e-learning for surgical training. Br J Surg. 2016;103:1428-37.

5. Tarpada S, Morris M, Burton D. E-learning in orthopedic surgery training: a systematic review. J Orthop. 2016;13(4):425-30.

6. Cook D, Garside S, Levinson A, et al. What do we mean by webbased learning? A systematic review of the variability of interventions. Med Educ. 2010;44:765-74.

7. Dong C, Goh P. Twelve tips for the effective use of videos in medical education. Med Teach. 2015;37:140-5.

8. Atlantis E, Cheema B. Effect of audience response system technology on learning outcomes in health students and professionals: an updated systematic review. Int J Evid Based Healthc. 2015;13:3-8.

9. Grzeskowiak L, Thomas A, To J, et al. Enhancing continued educational activities using audience response systems: a single-blind controlled trial. J Contin Educ Heal Prof. 2015;35(1):38-45.

10. Nelson C, Hartling L, Campbell S, et al. The effects of audience response systems on learning outcomes in health professions education. A BEME systematic review: BEME Guide No. 21. Med Teach. 2012;34:e386-405.

11. Rackshasbhuvankar A, Patole S. Benefits of simulation based training for neonatal resuscitation training: a systematic review. Resuscitation. 2014;85:1320-3.

12. Cook D, Brydges R, Hamstra S, et al. Comparative effectiveness of technology-enhanced simulation versus other instructional methods: a systematic review and meta-analysis. Simul Healthc. 2012;7:308-20.

13. Mundell W, Kennedy C, Szostek J, et al. Simulation technology for resuscitation training: a systematic review and meta-analysis. Resuscitation. 2013;84:1174-83.

14. Prgomet M, Georgiou A, Westbrook J. The impact of mobile handheld technology on hospital physicians' work practices and patient care: a systematic review. J Am Med Inform Assoc. 2009;16(6): 792-801.

15. Mosa A, Yoo I, Sheets L. A systematic review of healthcare applications for smartphones. BMC Med Inform Decis Mak. 2012;12: https://doi.org/10.1186/1472-6947-12-67.

16. Cheston $\mathrm{C}$, Flickinger T, Chisolm M. Social media use in medical education: a systematic review. Acad Med. 2013;88(6):893-901.

17. Pinilla S, Weckbach L, Alig S, Bauer H, Noerenberg D, Singer $\mathrm{K}$, et al. Blogging medical students: a qualitative analysis. GMS Z Med Ausbild. 2013;30(1):Doc9. https://doi.org/10.3205/ zma000852.

18. Hollinderbäumer A, Hartz T, and Ückert F. Education 2.0-how has social media and Web 2.0 been integrated into medical education? A systematic literature review. GMS Z Med Ausbil. 2013;30(1). https://doi.org/10.3205/zma000857.

19. The Psychiatry Milestone Project. Accreditation Council for Graduate Medical Education. 2015. https://www.acgme.org/ Portals/0/PDFs/Milestones/PsychiatryMilestones.pdf?ver=201511-06-120520-753. Accessed 9 Jan 2018. 